\title{
Machine Learning Methods to Investigate Drug Delivery in Infusion Pump
}

\author{
${ }^{1} \mathrm{~J}$. V. Alamelu, ${ }^{2} \mathrm{~A}$. Mythili \\ ${ }^{1}$ SENSE, Vellore Institute of Technology, Vellore and \\ EIE, M.S.Ramaiah Institute of Technology, Bangalore, \\ email: jvalamelu@gmail.com ; \\ ${ }^{2}$ SENSE, Vellore Institute of Technology, Vellore, \\ email: mythili.asaithambi@vit.ac.in, INDIA
}

Received: May 28, 2021. Revised: November 21, 2021. Accepted: December 10, 2021. Published: January 3, 2022.

\begin{abstract}
In the current scenario, usage of the smart medical pump is predominant in the medical field. The precise drug dosage, flow accuracy should be maintained to increase the performance of an infusion pump. In this work, an attempt has been made to predict and control the speed of the infusion pump for suitable infusion flowrate using machine learning technique and Linear Quadratic Gaussian (LQG) controller. The data for this study is considered from the publicly available online database, electronic Medicines Compendium (eMC). The speed of the infusion pump has been calculated using the drug dosage and flow rate for two different drugs. The prediction of infusion pump speed is achieved using Linear regression with Principal Component analysis (PCR) and Support Vector Machine Regression (SVR). The performance of the prediction schemes is evaluated using standard metrics. To validate the optimal control of the predicted speed, two different medical graded motors are considered. Further, the optimal control of the pump speed is investigated using Proportional-Integral-Derivative (PID), Linear Quadratic Regulator (LQR), and LQG controllers for its stability criteria. The prediction of the pump speed using regression models PCR, SVR has been verified and then the transient response analysis with rise time, settling time for both the motors have been examined. Results demonstrate that the LQG optimal control strategy achieves fast rise time, settling time of motor 1 with $0.653 \mathrm{~s}, 1.15 \mathrm{~s}$, and 0.22 , $0.392 \mathrm{~s}$ for motor 2 respectively.
\end{abstract}

Keywords- Machine learning technique, Flow rate, Infusion pump, Prediction, Regression

\section{INTRODUCTION}

Continuous and intravenous infusion of the drug, fluid, nutritional substances, antibiotics, insulin is managed by smart infusion pumps. A rapid change is happening in the medical device industry with the design of smart medical infusion pumps [1]. The smart medical devices used by health care providers are aimed to diagnose and treat patients as per the conditions of patients accurately [2],[3]. Clinical studies on infusion pumps have been transformed for several decades by using different designs with manual, semi-automated, and automated systems. In smart infusion pumps, the drug dosage to be infused, flow rate settings are controlled manually or automatically the closed loop drug infusion concepts are reported [4], [5]. The dosing errors are more in manually programmed pumps as well as in syringe pumps and misleading of drug delivery, flow rate, miscalculations have been discussed [6]. Minimization of errors in smart infusion pumps has been incorporated with drug libraries in the pre-programming environment, warnings, soft alarms, dosage limits [7]. The implementation is carried out by the manufacturer based on the Food and Drug Administration (FDA) guidelines for the infusion pumps. Even though the software, drug delivery guidelines are existing, the functionality and pumping mechanism differs depending on the types of infusion pumps [8]. Various kinds of infusion pumps are available, which include large volume pumps, syringe pumps, patient controlled analgesia, elastomeric pumps, enteral, and insulin pumps[9]. These pumps are used effectively in clinics, hospitals, and homes in which the pumping mechanism differs depending on the requirement to control the flow of the fluid to be delivered. The delivery of the fluid varies for each type of pump used and performance varies with respect to delay and flow rate is indicated [10]. 
The performance of the infusion pumps for effective drug and fluid delivery has been analyzed statistically using prediction models [11]. Besides, the impact of flow rate based on the carrier fluid has been investigated [12]. Prediction models such as ANN, ANFIS, PCR, SVR are implemented in the field of medical devices [13]. These techniques are addressed by many researchers for a wide range of applications which is not only limited to the medical field. Research on infusion pumps are carried out based on the valves that obstruct the maximum flow rate in intravenous infusion procedures when administered under gravity [14]. Drop in the flow rate around 19 to $38 \%$ is observed in $16 \mathrm{G}$ Canula when compared with $20 \mathrm{G}$ cannula. Westcott Sae-flow valve is used to produce a lower reduction in flow rate [15]. The flow rate accuracy is achieved with low flow rated syringe pumps based on the pressure applied [16]. The optimum flow control and high precision are vital for all the types of fluid and drug to be infused [17]. Further, the performance of medical pumps is important in real time conditions to administer the dead space [18].

Design of portable lightweight infusion pump with miniaturized components with battery powered motors are suitable to treat patients in clinical and home environment. Recent studies on decision making of the infusion pump speed based on the flow of the fluid using Fuzzy logic techniques [19]. The infusion pump control parameters such as dead volume, lag time, prediction accuracy, optimal speed control of the motor are essential to infuse the required volume of the drug and fluid. The electromechanical infusion pumps are employed with motors such as DC and stepper [20]. Statistical, calibration methods are used to obtain precise and accurate delivery for a range of flow rates with different types of pumps. Research on modeling and closed loop control systems for automatic control of anesthesia drugs is addressed [21], [22]. The automated control model for infusion pumps based on state space model as per pharmacokinetic representation is analyzed [23]. The control strategies are needed to manage the discontinuous and inaccurate flow of actuating mechanisms. LQG design method for anesthesia devices with frequency domain analysis is verified to obtain stability [24]. Many research is carried out using PID, LQR, and LQG for infusion pumps and electric motors [25]. Optimization and tuning techniques in the control system perspective have been examined for electric motors [26], [27].

The objective of this study is to predict and control the speed of medical graded infusion pumps. In this work, prediction techniques are used to predict pump speed for the corresponding drug dosage, flow rate. Further, to achieve the optimal speed control for infusion pumps, two medical graded electric motors have been considered and verified with their performance by employing control algorithms.

\section{MATERIALS AND METHODS}

The proposed workflow shown in Fig. 1 provides the process of prediction and controlling of infusion pump speed and its performance analysis. This study includes identifying the pump parameters, predicting the pump speed, and performance analysis using different control strategies.

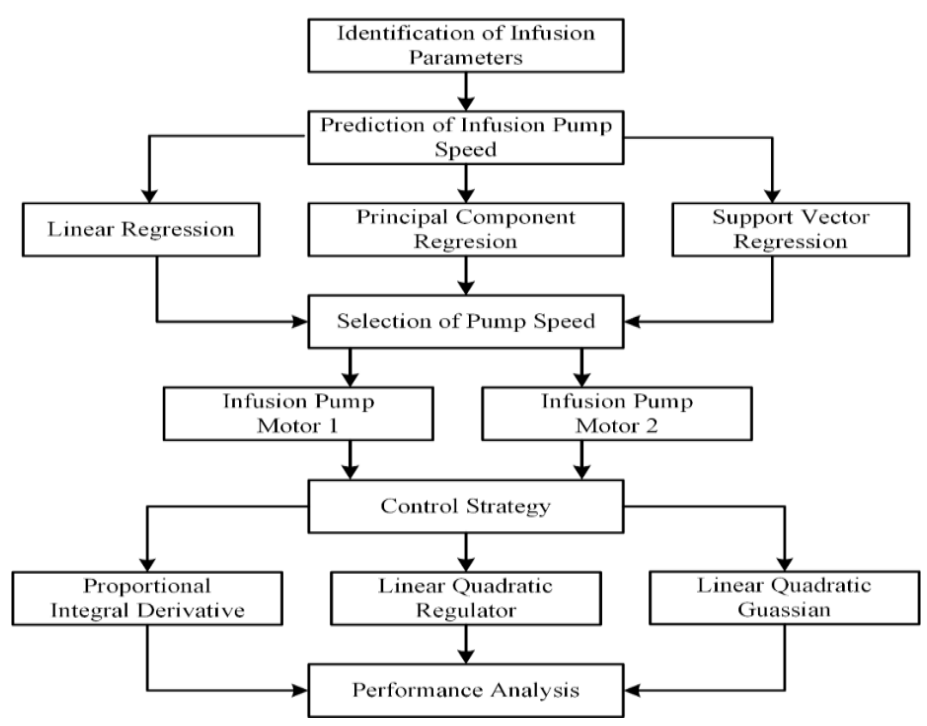

Fig.1. Proposed workflow

\section{A. Infusion pump parameters}

The data set of 100 infusion flowrates for different drugs for the patient with a mean weight of $65 \pm 12.9 \mathrm{~kg}$ has been obtained from a publicly available database, electronic Medicines Compendium (eMC) website (September 2020). The drug dosage, infusion pump speed, method of administration, and infusion flow rate are considered for this study. The important parameter to be addressed for an infusion pump is infusion flowrate and its speed. The key attributes of infusion flow rate $\left(Q_{d}\right)$ are drug dosage, drip rate, carrier flow $\left(\mathrm{Q}_{\mathrm{c}}\right)$. The total flow rate $\left(\mathrm{Q}_{\mathrm{t}}\right.$ is calculated as, $\mathrm{Q}_{\mathrm{t}}=\mathrm{Q}_{\mathrm{d}}+$ $\mathrm{Q}_{\mathrm{c}}$ [28]. For an infusion pump, its flow rate is based on the speed of the motor $(\theta)$ and it is related as, $Q_{t}=k \times \theta$ where $\mathrm{k}$ is the proportionality constant. Further, the speed of the motor $\theta=\mathrm{Q}_{\mathrm{t}} \times 10 \mathrm{rev} / \mathrm{ml} / 60 \mathrm{~min}$ per $\mathrm{hr}$ has been determined as per the manufacturer specification. The calculation varies for the number of gears in the motor and it physical interface with the pump as per the number of revolutions.

B. Prediction of the speed of infusion pump

Machine learning techniques are widely used for prediction and classification. In this work, prediction techniques are focused. In smart infusion pumps, 
parameter settings for different drug dosages should be administered. Especially in multiple infusion scenario, flow rate error has to be minimum. The flow rate error is based on how quickly the pump is actuated. This leads in optimal control of speed of infusion pump. To achieve the optimum speed of infusion pump, the prediction of the required speed corresponding to the drug flow rate for the pump's motor is principal. Prediction of pump speed for intravenous, continuous, subcutaneous infusion of drug flow rates has to be determined. Prediction algorithms are needed to reduce the adverse effect that will impact on patients. In this work the prediction of speed has been determined using machine learning technique such as classic linear regression, PCR, SVR for two different drug data set. The predictive model generates the future results once data is collected, trained, and analyzed.

\section{Regression methods}

Regression analysis is a statistical analysis method, determine the quantitative relationship between two or more variables [29].

In general, the multiple regression is formulated as

$$
y=\beta_{0}+\beta_{1} x_{1}+\cdots \cdot \varepsilon
$$

wherein $\mathrm{y}, \mathrm{x}$ is the dependent, independent variable, $\beta$ is the coefficient parameter and $\varepsilon$ is the error or residuals. By providing the $x_{i}$ for the regression model, the predicted values are estimated and it is given by $\hat{y}$. The fitted regression function is given as

$$
\hat{y}=\sum b_{k} f_{k}\left(x_{i} \ldots\right), i=1,2 \ldots n
$$

where $b_{k}$ is the fitted coefficients. The mean square difference between the $\hat{y}-y$ is identified, minimized by estimating $b_{k}$.

The drug dosage rate was the input variable and tested with linear regression, the prediction fit was found to be multicollinear. Multicollinearity is a statistical phenomenon where more than two predictor variables in a multiple regression model are highly correlated. In this situation, the coefficient estimates change erratically for small changes in the model or the data which in-turn affects the calculations of individual predictors. Thus, a multiple regression model with correlated predictors indicates the overall accuracy of the outcome variable predictions. On the other hand, valid results would not be obtained with individual predictor and also with redundant predictors.

\section{Principal component regression}

Principal Component Regression (PCR) is a technique, utilizes principal component analysis by regression coefficients estimation [30]. PCR is implemented to overcome issues that arises when the exploratory variables are close to being colinear. In PCR, instead of regressing the independent variables (the regressors) on the dependent variable directly, the principal components of the independent variables are used. To estimate, subset of the principal components in the regression is used.

In PCR the regression coefficient $\hat{\beta}$ is estimated as given in equations 3,4 .

$$
\widehat{\beta}=\left(x^{\prime} x\right)^{-1} x^{\prime} y
$$

(3)

Mathematically, $\quad R=x^{\prime} x=P D P^{\prime}=Z^{\prime} Z$ (4)

where, $\mathrm{R}$ is the correlation matrix of independent variables, $\mathrm{D}$ is a diagonal matrix, $\mathrm{Z}$ is data matrix, $\mathrm{P}$ is orthogonal $\left(P P^{\prime}=1\right)$. The regression with principal component has to be done as given below:

1. PCA on $\mathrm{x}$ has to be performed and $\mathrm{z}$ will be obtained which is a new variable with weighted averages

2. Regression fit $\mathrm{y}$ has to be identified with $\mathrm{Z}$ by

$$
\text { estimating A were in } A=P^{y} B, B=P A
$$

PCR is a regression method that can be divided into three steps: the first step is to execute a principal components analysis on the table of the explanatory variables. The second step is to impose an ordinary least squares regression (linear regression) on the selected components: the factors that are most correlated with the dependent variable will be selected. Finally, the parameters of the model are computed for the selected explanatory variables.

\section{Support Vector Regression}

Support Vector Machine (SVM) is a typical supervised learning method in machine learning. It is a classifier and performs regression is termed as SVR [31]. SVR is based on the concept of minimization of structural risk hypothesis, which lessens both empirical risk and the confidence interval of the learning machine. This leads to producing better generalization capability, recognize the adequate error ranges for any given model, and find hyperplane to fit the data. SVR has an objective function that helps in minimizing the coefficient vector.

In general, the basic function for the statistical learning process in SVM is given in the equation below.

$$
y=f(x)=\sum_{j=1}^{M} w_{i} x_{j}+b
$$

where $\mathrm{y}$ is the independent variable, $\mathrm{w}$ is the weighted coefficient for sum of $\mathrm{M}, \mathrm{b}$ is the coefficient parameter.

$$
f(x)=\left[\begin{array}{l}
w \\
b
\end{array}\right]^{T}\left[\begin{array}{l}
x \\
1
\end{array}\right]=w^{T} x+b
$$

The objective function minimization is needed to obtain the desired predict outputs and is given as

$$
\min (w)=\frac{1}{2}\left\|w^{2}\right\|
$$

The objective function minimization is important and the empirical error is reduced based on that. The difference between the predicted and actual value is within the threshold when loss is zero value. For other 
values, the loss will be equal to the difference between the predicted value and the radius of hyperplane. To perform this, Lagrange multipliers and kernel function are solved. Appropriate kernel allows the data to become separable in the feature space, even though data is non separable in the original input space.

\section{PERFORMANCE EVALUATION OF THE PREDICTION MODELS}

Data processing is an important criterion to evaluate the performance of an infusion pump. The infusion pump working is purely dependent on the type of infusion pump, manufacturer and drug dosage protocol followed by the physician, nurses. The posology, flow rate calculations are made as per the nurses' guide. In electronic Medical Compendium (eMC), the dosage for different drugs is specified in $\mu \mathrm{g} / \mathrm{kg} / \mathrm{min}$ and $\mathrm{mg} / \mathrm{hr}$, the flow rate will be in terms of units $/ \mathrm{hr}$ or $\mathrm{ml} / \mathrm{hr}$. As per the nursing calculations, the drip rate, flow rate for each drug has been converted and the data set has been prepared.

The output variable which is the speed of the pump is calculated. The number of revolutions varies based on the type of motor employed in infusion pumps which are designed as per the medical device and its usage. The prediction of this variable has been analysed for its accuracy using the prediction model considered in this current work. The statistical metrics utilized to identify the performance of LR, PCR, and SVR approach are mean square error (MSE), root mean square error (RMSE), mean absolute error (MAE), and coefficient of determination $\left(\mathrm{R}^{2}\right)$ [31]. MSE, RMSE, and MAE are used to identify the prediction error of the predictive models. Prediction accuracy is evaluated using the $\mathrm{R}^{2}$ value.

The statistical metrics are:

$$
\begin{aligned}
& \text { 1. } M S E=\frac{1}{n} \sum_{i=1}^{n}\left(x_{i}-y_{i}\right)^{2} \\
& \text { 2. } R M S E=\sqrt{\frac{1}{n} \sum_{i=1}^{n}\left(x_{i}-y_{i}\right)^{2}} \\
& \text { 3. } M A E=\frac{1}{n} \sum_{i=1}^{n}\left(x_{i}-y_{i}\right) \\
& \text { 4. } R^{2}=\frac{n\left(\sum x y\right)-\sum x \sum y}{\sqrt{\left[n \sum x^{2}-\left(\sum x\right)^{2}\right]\left[n \sum y^{2}-\left(\sum y\right)^{2}\right]}}
\end{aligned}
$$

where the $x_{\mathrm{i}}$ is the actual output variable, $y_{\mathrm{i}}$ is the predicted output variable and $\mathrm{n}$ is the number of samples in the testing set. The performance of the infusion pump exclusively relies on its speed, dosage, and flow rate.

\section{CONTROL SYSTEM STRATEGY FOR INFUSION PUMP}

The selection of infusion pump optimal control can be achieved with different types of electromechanical motors. The rotational speed of these motors decides the speed of the pump based on the flow rate and the revolutions. The input to these controllers is from the predicted speed obtained from the predictive model and time response analysis of the pump control system which is employed with micromotors has been verified. In this study, the micromotor of an infusion has been chosen as per the manufacturer specification of medical pumps and analysed. Two specific pumps motor had been considered with required electrical, mechanical parameters and are used to derive the transfer function of the infusion pump. To employ this, the control system for an infusion pump actuation has become essential. The implementation of control algorithms such as PID controller, LQR, and LQG has been carried out. The identification of the stability, steady-state response, and pole replacement for the control system design have been executed to obtain quick rise time and fast settling time.

\section{A. PID controller}

PID is the widely used controller which is used to obtain the required control parameters by finding the error signal for a system. In this work, the transfer function for the actuating part of the infusion pump is given in the equation below

$$
Q(s)=K * \frac{\omega(s)}{\Theta(s)}=K * \frac{K_{T}}{\left(L_{a}(s)+R_{a}\right)(j s+B)+\left(K_{b} K_{T}\right)}
$$

where $\mathrm{Q}(\mathrm{s})$ is the flowrate which is obtained as the product of gain factor $\mathrm{K}$ and $\omega(s)$ is the speed of electric motor. In equation (12), Torque constant $\mathrm{K}_{\mathrm{T}}$, Armature inductance $\mathrm{L}_{\mathrm{a}}$, Armature resistance $\mathrm{R}_{\mathrm{a}}$, Rotating inertial measurement of motor bearing J, e.m.f constant $\mathrm{K}_{\mathrm{b}}$, Friction constant $\mathrm{B}$ for the DC motor is given. The transfer function of the micromotor with its mechanical and electrical parameters has been considered to define the transfer function of the micromotor. To obtain closed control for the infusion pump, the predicted speed has been given as input, and using controller strategy the speed control for micromotor has been achieved. The equation below is shown with the controller output $u(t)$ when incorporated with PID controller.

$$
u(t)=K_{p} e(t)+K i \int_{0}^{t} e(t) d(t)+K_{d} \frac{d e(t)}{d t}
$$

Where $\mathrm{K}_{\mathrm{p}}, \mathrm{K}_{\mathrm{i}}, \mathrm{K}_{\mathrm{d}}$ are the gain constants for PID and $\mathrm{e}(\mathrm{t})$ is the error. The gain values of the PID have been tuned using the traditional Zeigler-Nichols method and for better optimized results optimizer such as PSO can be implemented [32]. 


\section{B. LQR controller}

The state space representation for the infusion pump motor is designed. The objective of implementing LQR is to obtain optimized speed control for infusion pump motors based on the predicted speed. In LQR, R and Q matrices determine values for closed loop control of pump motors. The $\mathrm{R}$ and $\mathrm{Q}$ values has to be trial and error manner. The range of values selected for $\mathrm{Q}$ relied on the different ranges of the unity matrix. Let, the cost function $\mathrm{J}$ is defined as

$$
I=\int_{0}^{\infty}\left(x^{t} Q x+u^{T} R u\right) d t
$$

The term linear quadratic refers to the linear system dynamics, the quadratic cost function, and the gain vector $\mathrm{K}$, which is found for cost function minimization. The optimum values for the state feedback matrix has been determined based on the state-space model and Ricatti equation for the LQR [33] is given as

$$
A^{T} P+P A-P B R^{-1}-P B R^{-1} B^{T} P+Q=0
$$

C. $L Q G$ controller

Linear Quadratic Gaussian is a classic optimal control strategy that has the ability to handle noise in the system. Certainly, the smart pump's accuracy will have noise disturbances and can be handled with LQG [34]. The linear quadratic regulator which is an optimal controller is combined with Kalman filter to manage external noise $\mathrm{w}$ and $\mathrm{v}$. the controller output $\mathrm{u}$ has to be obtained based on the minimization of the cost function,

$$
I(u)=\frac{1}{2} \int_{0}^{\infty}\left(x^{T}+2 x^{T} N u+u^{T} R u\right) d t
$$

where, $\mathrm{N}$ is the value between control and the regulation performance. The state feedback matrix, $u(t)$ is given as $\mathrm{u}(\mathrm{t})=-\mathrm{k}^{*} \mathrm{x}(\mathrm{t})$, is required to minimize $\mathrm{J}(\mathrm{u})$.

Further, k, which is the gain matrix has to be solved algebraically using Riccati equation which is is given as $A^{T} s+s A-(s B+N) R^{-1}\left(B^{T} s+N^{T}\right)+Q=0$

(17)

where $\mathrm{s}$ is a matrix and $\mathrm{k}$ is obtained from $\mathrm{s}$. The equation is expressed as,

$K=R^{-1}\left(B^{T} s+N^{T}\right)$

Further, the Kalman gain has to be determined for the infusion pump. In general, the process noise $\mathrm{Q}$ and measurement noise $\mathrm{R}$ is obtained as,

$$
\mathrm{Q}=\mathrm{E}\left[\mathrm{ww}^{\mathrm{T}}\right] \text { and } \mathrm{R}=\mathrm{E}\left[\mathrm{ww}^{\mathrm{T}}\right]
$$

Further, the Kalman gain, covariance matrix will be determined and the response plot for the infusion pump will be plotted to verify the control response parameters $t_{r}, t_{s}$, and stability. The design of the transfer function has been modified and verified for two different micromotor specifications with 0651 and 1224A012 series as used in medical devices. The transfer function obtained has been verified for its stability with respect to the pole position and thereby pole replacement is accomplished to get a stable output response. The linear quadratic controllers aim to reduce the quadratic function with state feedback and performance of the system is improved.

\section{RESULTS}

The performance analysis of the prediction models has been analyzed for two different drugs for a subject having a mean weight of $65 \pm 12.9 \mathrm{~kg}$. Prediction of infusion pump speed is carried out for both the drugs are presented in Table 1. It is observed that the predictive model's PCR and SVR show a better fit as the statistical parameters are found to be in the ideal range. The prediction error is analyzed using RMSE, MSE, MAE,

\begin{tabular}{|c|c|c|c|c|c|c|c|c|}
\hline \multirow{2}{*}{$\begin{array}{c}\text { Prediction } \\
\text { model }\end{array}$} & \multicolumn{5}{|c|}{ Drug 1} & \multicolumn{3}{|c|}{ Drug 2} \\
\hline & RMSE & $\mathbf{R}^{2}$ & MSE & MAE & RMSE & $\mathbf{R}^{2}$ & MSE & MAE \\
\hline PCR & 0.0242 & 1 & 0.00586 & 0.01169 & 0.0045 & 1 & $2.04 \mathrm{e}-05$ & 0.0019 \\
\hline SVR & 0.4419 & 1 & 0.195 & 0.39 & 0.154 & 0.99 & 0.024551 & 0.13494 \\
\hline
\end{tabular}
and $\mathrm{R}^{2}$.

Table 1 Performance comparison of prediction models for predicted infusion pump speed

The correlation between the independent variables and infusion pump speed is verified for two different drugs using LR, PCR and SVR. The correlation coefficient in PCR and SVR is found to be high as 1 . The prediction parameters found to be collinear when analyzed using LR for the input variables drug dosage, the flow rate. The predicted and residual response for infusion pump speed for different drug dosages is shown in Fig. 2 and Fig. 3.

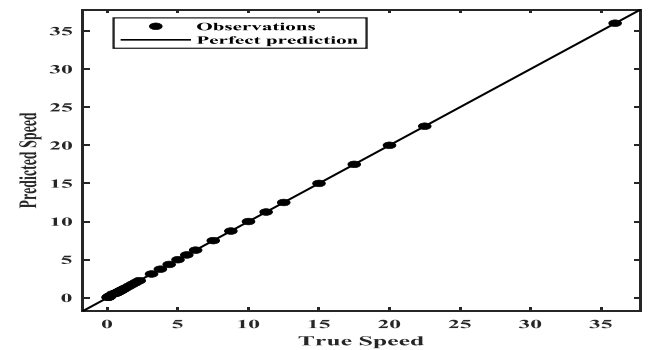

(a)

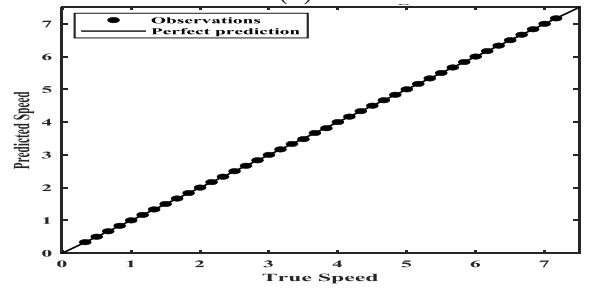

(b)

Fig.2. Linear regression analysis with PCR between actual and predicted speed for (a) drug1 and (b) drug2 


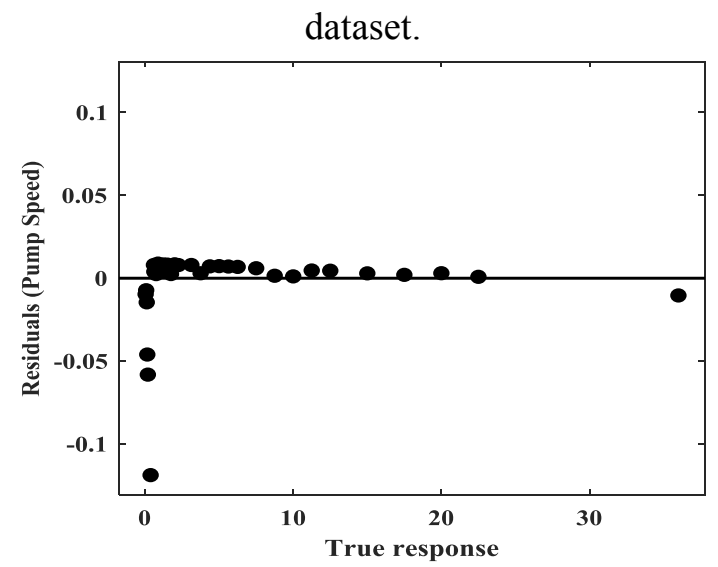

(a)

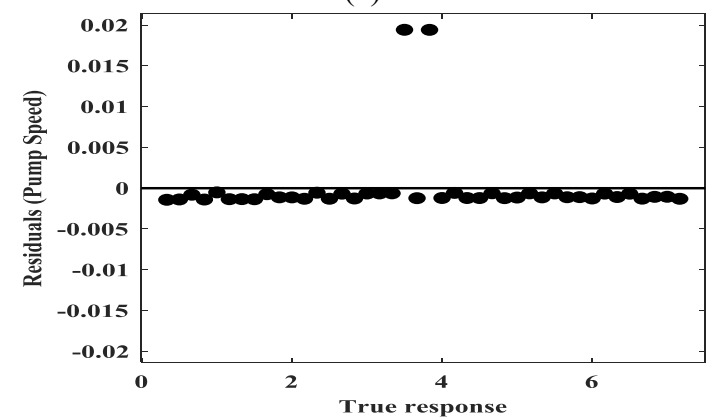

(b)

Fig.3. The residual response of actual and predicted speed using PCR for (a) drug1 and (b) drug2 datasets.

Table 2 Performance analysis of two infusion pump

\begin{tabular}{|l|l|l|l|l|l|l|}
\hline & \multicolumn{3}{|l|}{ Infusion Pump 1 } & \multicolumn{3}{l|}{ Infusion Pump 2 } \\
\cline { 2 - 7 } & LQR & $\begin{array}{l}\text { LQG } \\
\text { (with } \\
\text { noise) }\end{array}$ & PID & LQR & $\begin{array}{l}\text { LQG } \\
\text { (with } \\
\text { noise) }\end{array}$ & PID \\
& & & & & & \\
\hline \\
\hline $\mathrm{t}_{\mathrm{r}}(\mathrm{sec})$ & 0.608 & 0.653 & 12.4 & 0.000616 & 0.22 & 5.5 \\
\hline $\mathrm{t}_{\mathrm{s}}(\mathrm{sec})$ & 1.04 & 1.15 & 19.3 & 0.00193 & 0.392 & 0.9 \\
\hline
\end{tabular}

The predicted speed of two different pumps is verified with PID, LQR, LQG control algorithms, and the dynamic performance parameter rise time $\left(t_{r}\right)$, settling time $\left(t_{s}\right)$ are shown in table 2 . The transient response of LQG is shown in Fig. 4 for both pumps.

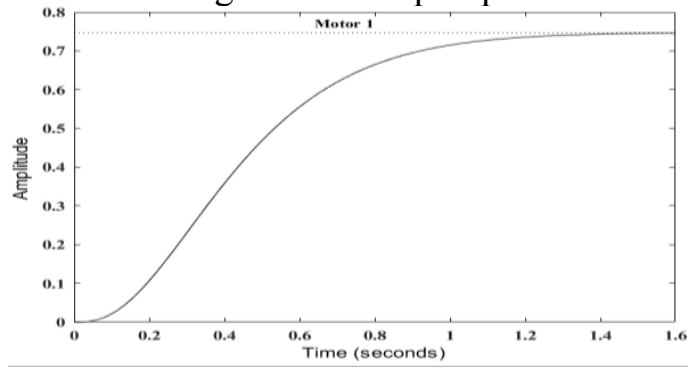

(a)

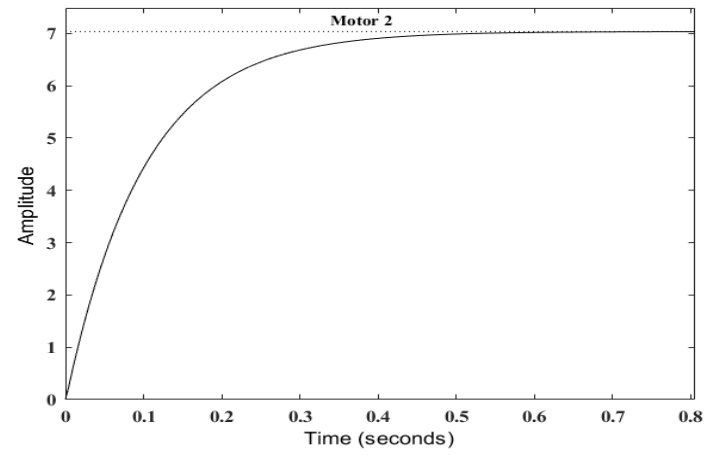

(b)

Fig. 4. Transient response for (a) pump motor1 and (b) pump motor2 with LQG controller.

\section{DISCUSSION}

Every drop of the fluid and drug to the patient is crucial. Prediction of speed and control of the pump plays a vital role in smart infusion systems. In this study, the infusion pump speed is predicted using LR, PCR, and SVR models. The performance evaluation of the predictive models is carried out with error indicator measures MSE, RMSE, MAE, and $\mathrm{R}^{2}$. The prediction algorithms tend to reduce the errors to yield high prediction accuracy. The prediction error for the PCR model is lower than that of the SVR model. The RMSE value of the PCR model is observed to be less with 0.0045 for drug2 and 0.0242 for drug1 compared to the RMSE of SVR model. The correlation coefficient indicates the better predictive performance of predictive models and a linear and high degree of correlation $\left(\mathrm{R}^{2}=1\right)$ is observed. Residual analysis for predictive models is implemented and the residues are found to be around 0 to achieve better prediction. The optimal speed control for two medical graded electric motors is verified with control strategies such as PID, LQR, and LQG. The transfer function of the controller is employed with pole replacement to obtain optimal control. The control schemes have been validated with rise time and settling time. Further, the optimal control techniques are achieved with a rise time of $0.653 \mathrm{sec}, 0.22 \mathrm{sec}$, and settling time of $1.15 \mathrm{sec}, 0.392 \mathrm{sec}$ for both the pumps respectively. The values obtained by using LQR are better than the traditional PID controller and when noise is prominent, LQG is preferred. The current study can be further validated with an infusion pump experimental setup by employing the proposed prediction and control models.

\section{CONCLUSION}

The performance of an infusion pump depends on the prediction and control of the pump speed, optimum flow rate. These parameters influence dead time and lag time for an infusion pump for different types of drugs. In this study, the prediction and control 
of the infusion pump for the corresponding drug dosage, the flow rate has been carried out. To achieve this LR, PCR, SVR regression models have been used. The predictive models are evaluated for accuracy and the required optimum pump motor parameters are given as setpoints to the controller. PID controller, LQR, and LQG controller have been implemented to obtain optimal control. The transient response analysis of the system has been examined to obtain required rise time, settling time for different control strategies. The response analysis shows that the LQG controller results with fast rise and settling time as required for the infusion pump. Hence, the study proves that the prediction and control of pump speed using regression models, optimal controllers are necessary to provide fast infusion of the drug to the patients with better accuracy.

\section{REFERENCES}

[1] D. Guelig et al., "Design of a novel, adjustable flow rate, reusable, electricity-free, low-cost syringe infusion pump," J. Med. Devices, Trans. ASME, vol. 11, no. 4, pp. 1-6, 2017, doi: 10.1115/1.4037935.

[2] J. Hughes et al., "Infusion pump-mediated mechanical hemolysis in pediatric patients," Ann. Clin. Lab. Sci., vol. 45, no. 2, pp. 140-147, 2015.

[3] U. R. Kim, R. A. Peterfreund, and M. A. Lovich, "Drug infusion systems: Technologies, performance, and pitfalls," Anesth. Analg., vol. 124, no. 5, pp. 1493-1505, 2017, doi: 10.1213/ANE.0000000000001707.

[4] C. H. Ting, R. H. Arnott, D. A. Linkens, and A. Angel, "Migrating from target-controlled infusion to closedloop control in general anaesthesia," Comput. Methods Programs Biomed., vol. 75, no. 2, pp. 127-139, 2004, doi: 10.1016/j.cmpb.2003.11.005.

[5] M. J. Parker et al., "Computer Control of Drug Delivery by Continuous Intravenous Infusion," Anesthesiology, vol. 122, no. 3, pp. 647-658, 2015, doi: 10.1097/aln.0000000000000519.

[6] R. A. Snijder, M. K. Konings, A. van den Hoogen, and A. M. D. E. Timmerman, "Impact of Physical Parameters on Dosing Errors due to a Syringe Exchange in Multi-Infusion Therapy," Pharm. Technol. Hosp. Pharm., vol. 2, no. 2, pp. 85-96, 2017, doi: 10.1515/pthp-2017-0002.

[7] A. Rajkomar and A. Blandford, "Understanding infusion administration in the ICU through Distributed Cognition," J. Biomed. Inform., vol. 45, no. 3, pp. 580-590, 2012, doi: 10.1016/j.jbi.2012.02.003.

[8] F. Engbers, "Pump pitfalls and practicalities," Total Intraven. Anesth. Target Control. Infusions, pp. 329340, 2017, doi: 10.1007/978-3-319-47609-4.

[9] R. A. Peterfreund and J. H. Philip, "Critical parameters in drug delivery by intravenous infusion," Expert Opin. Drug Deliv., vol. 10, no. 8, pp. 10951108, 2013, doi: 10.1517/17425247.2013.785519.

[10] J. G. Hobbs et al., "Flow rate accuracy of ambulatory elastomeric and electronic infusion pumps when exposed to height and back pressures experienced during home infusion therapy," Expert Rev. Med. Devices, vol. 16, no. 8, pp. 735-742, 2019, doi: 10.1080/17434440.2019.1632187.

[11] J. J. Min, D. K. Kim, K. Y. Hong, J. W. Choi, and K. Y. Choi, "Comparison of operator workloads associated with the single-unit Anyfusion ${ }^{\circledR}$ pump and the changeover from a syringe pump to an infusion pump," J. Korean Med. Sci., vol. 34, no. 49, pp. 1-9, 2019, doi: 10.3346/jkms.2019.34.e314.

[12] Z. C. Madson, S. Vangala, G. T. Sund, and J. A. Lin, "Does carrier fluid reduce low flow drug infusion error from syringe size?," World J. Clin. Pediatr., vol. 9, no. 2, pp. 17-28, 2020, doi: 10.5409/wjcp.v9.i2.17.

[13] I. M. El-Hasnony, S. I. Barakat, and R. R. Mostafa, "Optimized ANFIS Model Using Hybrid Metaheuristic Algorithms for Parkinson's Disease Prediction in IoT Environment," IEEE Access, vol. 8, pp. 119252-119270, 2020, doi: 10.1109/ACCESS.2020.3005614.

[14] J. M. Hall and F. L. Roberts, "An investigation into the reduction in flow rate of intravenous fluid by antireflux valves," Anaesthesia, vol. 60, no. 8, pp. $797-800, \quad 2005, \quad$ doi: $\quad 10.1111 /$ j.13652044.2005.04239.x.

[15] R. A. Snijder, M. K. Konings, P. Lucas, T. C. Egberts, and A. D. Timmerman, "Flow variability and its physical causes in infusion technology: A systematic review of in vitro measurement and modeling studies," Biomed. Tech., vol. 60, no. 4, pp. 277-300, 2015, doi: 10.1515/bmt-2014-0148.

[16] M. Batliner, M. Weiss, S. A. Dual, B. Grass, M. Meboldt, and M. Schmid Daners, "Evaluation of a novel flow-controlled syringe infusion pump for precise and continuous drug delivery at low flow rates: a laboratory study," Anaesthesia, vol. 74, no. 11, pp. 1425-1431, 2019, doi: 10.1111/anae.14784.

[17] K. S. Tee, M. S. Saripan, H. Y. Yap, and C. F. Soon, "Development of a Mechatronic Syringe Pump to Control Fluid Flow in a Microfluidic Device Based on Polyimide Film," IOP Conf. Ser. Mater. Sci. Eng., vol. 226, no. 1, 2017, doi: 10.1088/1757$899 X / 226 / 1 / 012031$.

[18] A. Foinard, B. Décaudin, C. Barthélémy, G. Lebuffe, B. Debaene, and P. Odou, "Impact of infusion set characteristics on the accuracy of patient-controlled morphine administration: A controlled in-vitro study," Anaesthesia, vol. 69, no. 2, pp. 131-136, 2014, doi: 10.1111/anae.12523.

[19] J. Li et al., "Intelligent infusion controller with a physiological information feedback function," Technol. Heal. Care, vol. 28, no. S1, pp. S37-S46, 2020, doi: 10.3233/THC-209005.

[20] Junaidi et al., "Flow Rate and Volume Control of Fluid Based on Arduino for Synthesis of Silver Nanowires," J. Phys. Conf. Ser., vol. 1338, no. 1, pp. 1-6, 2019, doi: 10.1088/1742-6596/1338/1/012018. 
[21] N. Simon, B. Décaudin, D. Lannoy, C. Barthélémy, M. Lemdani, and P. Odou, "Mathematical and physical model of gravity-fed infusion outflow: Application to soft-bag-packed solutions," Eur. J. Drug Metab. Pharmacokinet., vol. 36, no. 4, pp. 197-203, 2011, doi: 10.1007/s13318-011-0062-9.

[22] P. Pankhurst and Z. McGuinness Abdollahi, "Evaluation of a novel portable micro-pump and infusion system for drug delivery," Proc. Annu. Int. Conf. IEEE Eng. Med. Biol. Soc. EMBS, vol. 2016Octob, pp. 465-468, 2016, doi: 10.1109/EMBC.2016.7590740.

[23] I. Nașcu and E. N. Pistikopoulos, "Modeling, estimation and control of the anaesthesia process," Comput. Chem. Eng., vol. 107, pp. 318-332, 2017, doi: 10.1016/j.compchemeng.2017.02.016.

[24] A. K. Patra and P. K. Rout, "An automatic insulin infusion system based on LQG control technique," Int. J. Biomed. Eng. Technol., vol. 17, no. 3, pp. 252-275, 2015, doi: 10.1504/IJBET.2015.068109.

[25] M. A. Aravind, N. Saikumar, and N. S. Dinesh, "Optimal position control of a DC motor using LQG with EKF," 2017 Int. Conf. Mech. Syst. Control Eng. ICMSC 2017, no. 2, pp. 149-154, 2017, doi: 10.1109/ICMSC.2017.7959461.

[26] A. Madadi and M. M. Motlagh, "Optimal Control of DC motor using Grey Wolf Optimizer Algorithm," Tech. J. Eng. Appl., pp. 373-379, 2014.

[27] S. Howimanporn, S. Chookaew, and W. Sootkaneung, "Implementation of PSO Based Gain-Scheduled PID and LQR for DC Motor Control Using PLC and SCADA," 2018 Int. Conf. Control Robot. ICCR 2018, no. September, pp. 52-56, 2018, doi: 10.1109/ICCR.2018.8534485.

[28] J. Brindley, "Undertaking drug calculations for intravenous medicines and infusions," Nurs. Stand., vol. 32, no. 20, pp. 55-63, 2018, doi: 10.7748/ns.2018.e11029.

[29] M. Esmaeili, M. Osanloo, F. Rashidinejad, A. Aghajani Bazzazi, and M. Taji, "Multiple regression, ANN and ANFIS models for prediction of backbreak in the open pit blasting," Eng. Comput., vol. 30, no. 4, pp. 549-558, 2012, doi: 10.1007/s00366-012-0298-2.

[30] L. V Perez, "Principal Component Analysis to Address Multicollinearity," pp. 1-20, 2017.

[31] N. Guo et al., "Using improved support vector regression to predict the transmitted energy consumption data by distributed wireless sensor network," Eurasip J. Wirel. Commun. Netw., vol. 2020, no. 1, 2020, doi: 10.1186/s13638-020-01729-x.

[32] M. M. Sabir and J. A. Khan, "Optimal Design of PID Controller for the Speed Control of DC Motor by Using Metaheuristic Techniques," Adv. Artif. Neural Syst., vol. 2014, pp. 1-8, 2014, doi: $10.1155 / 2014 / 126317$.

[33] R. M. K. Hummadi and A. Hummadi, "Simulation of Optimal Speed Control for a Dc Motor Using Linear Quadratic Regulator," J. Eng., vol. 18, no. 3, pp. 340349, 2012.
[34] M. A. Aravind, N. Saikumar, N. S. Dinesh, and K. Rajanna, "Stability analysis and efficiency of EMPC for Type-1 systems," Int. J. Dyn. Control, vol. 7, no. 2, pp. 452-468, 2019, doi: 10.1007/s40435-018-0461-8.

\section{Sources of funding for research presented in a scientific article or scientific article itself \\ Report potential sources of funding if there is any \\ Creative Commons Attribution License 4.0 (Attribution 4.0 International, CC BY 4.0)}

This article is published under the terms of the Creative Commons Attribution License 4.0

https://creativecommons.org/licenses/by/4.0/deed.en_US 